\title{
BMJ Open Qualitative inquiry into barriers and facilitators to transforming primary care for lesbian, gay, bisexual and transgender people in US federally qualified health centres
}

Kelly W Gagnon (D) ,1,2,3 Lauren Bifulco (D) , ${ }^{2}$ Sarafina Robinson, ${ }^{2}$ Bruce Furness, ${ }^{4,5}$ Daniel Lentine, ${ }^{4}$ Daren Anderson ${ }^{2}$

To cite: Gagnon KW, Bifulco L, Robinson S, et al. Qualitative inquiry into barriers and facilitators to transforming primary care for lesbian, gay, bisexual and transgender people in US federally qualified health centres. BMJ Open 2022;12:e055884. doi:10.1136/ bmjopen-2021-055884

- Prepublication history and additional supplemental material for this paper are available online. To view these files, please visit the journal online (http://dx.doi.org/10.1136/ bmjopen-2021-055884)

Received 02 August 2021 Accepted 27 January 2022
Check for updates

(C) Author(s) (or their employer(s)) 2022. Re-use permitted under CC BY-NC. No commercial re-use. See rights and permissions. Published by BMJ.

For numbered affiliations see end of article.

Correspondence to

Kelly W Gagnon;

keg118@pitt.edu

\section{ABSTRACT}

Objectives Health systems must rapidly move knowledge into practice to address disparities impacting sexual and gender minority (SGM) patients. This qualitative study explores barriers and facilitators that arose during an initiative to improve care for SGM patients in federally qualified health centres (FQHCs) from the perspectives of FQHC staff.

Design Cross-sectional qualitative content analysis, using a general inductive approach, of secondary data from transcripts of intervention events offered to $\mathrm{FQHC}$ staff and semistructured interviews with staff and FQHC leadership during the intervention.

Setting 10 FQHCs from nine states in the USA.

Participants $\mathrm{FQHC}$ quality improvement (QI) and clinical care staff, and leaders at each FQHC.

Interventions The transforming care for lesbian, gay, bisexual and transgender people Ql initiative combined two evidence-based programmes, Learning Collaborative (LC) and Project Extension for Community Healthcare Outcomes (ECHO), to assist primary care health centres in developing capacity to identify SGM patients, monitor their health and care, and improve disparities.

Primary and secondary outcome measures The primary outcome was identification of barriers and facilitators to implementing initiatives to improve care for SGM patients. The secondary outcome was clarification of how intervention participants used Project ECHO sessions versus LC meetings to obtain information that influenced implementation of the initiative at their FQHC.

Results Barriers and facilitators mapped to two major themes: 'Clinical' (patients' health, wellness, and available treatment) and Health Systems and Institutional Culture (FQHC operations, and customs and social institutions within the FQHCs and in the external environment). Common 'Clinical' inquiries were for assistance with behavioural health, pre-exposure prophylaxis and transgender hormone therapy. Prevalent facilitators included workflow change and staff training, while adapting electronic health records for data collection, decision support and data extraction was the most prevalent barrier.
Strengths and limitations of this study

- The breadth of participants included in the study, from quality improvement staff and clinical providers to clinical leadership, provided a multistakeholder understanding of experienced barriers and facilitators.

- This study is novel in its exploration of the implementation of two evidence-based programmes to modify systems to improve population health.

- We analysed and triangulated secondary data from three sources: Project Extension for Community Healthcare Outcomes clinical case presentations, Learning Collaborative meetings and semistructured interviews with federally qualified health centres leadership and implementation teams, which provided a more holistic understanding of the implementation process.

- While inclusive of a wide range of health care staff, administrative staff and patient perspectives were not available.

Conclusions Project ECHO and LC provided complimentary forums to explore clinical and operational changes needed to improve care for SGM at FQHCs.

\section{INTRODUCTION}

Sexual and gender minorities (SGM) are at increased risk for poor physical and mental health outcomes ${ }^{1-4}$ and may have limited access to affirming, culturally competent healthcare. ${ }^{5}$ Medical providers, particularly those in primary care settings (where most routine care is provided), have limited knowledge and expertise in caring for SGM patients. ${ }^{6}$ Few health centres have adequate systems in place to capture critical data about patients' sexual orientation and gender identity $(\mathrm{SOGI})^{7-10}$; provide a comfortable, affirming environment that appropriately 
acknowledges patients' intersectional social identities ${ }^{11-14}$; or deliver evidence-based care for health conditions disproportionally impacting SGM.

'Transforming Primary Care for LGBT People' (Transforming LGBT Care: Though the acronym LGBT (lesbian, gay, bisexual and transgender) was used in the project title and the name of one of the intervention components, all SGM patients were included as part of the target population.) was a 1-year intervention for federally qualified health centres (FQHCs) aimed at improving primary care for SGM people. Methods and outcomes have been previously described. ${ }^{15}$ Briefly, the initiative aimed to help participants better align their primary care services with SGM patients' needs by: (1) educating clinical providers on SGM health disparities and (2) introducing SOGI data collection processes that allowed FQHCs to identify SGM patients and implement risk-based sexual history and sexually transmitted infection (STI) screenings. Transforming LGBT Care facilitated a $276.3 \%$ increase in number of patients with documented SOGI data across 10 FQHCs postintervention, and led to improvements in sexually transmitted disease screening for SGM patients and uptake of affirming training, practices, policies and systems. $^{15}$

To achieve these outcomes, Transforming LGBT Care offered a Project Extension for Community Healthcare Outcomes (Project ECHO) telehealth videoconference series for clinical knowledge sharing, and a quality improvement (QI) learning collaborative (LC) videoconference series focused on caring for SGM patients. Project ECHO is a telementoring and continuing education intervention that trains primary care providers (PCPs) in specific areas of specialty care to help overcome disparities in access to care. ${ }^{16-20}$ Transforming LGBT Care was one of the first interventions to utilise the Project ECHO model to address healthcare disparities for a specific population $^{21-23}$ (SGM) rather than a health condition. The simultaneous LC $^{24}$ was integrated to address synergistic health systems issues and to help FQHCs use QI strategies to design, test and implement sustainable processes and procedures to improve care for SGM individuals. ${ }^{2526}$ Specifically, the LC aimed to assist health centres through the process of developing protocols and systems to collect patients' SOGI data. SOGI data are crucial for population health management and is often difficult to collect because of the history of stigma, discomfort, and bias experienced by SGM patients. ${ }^{12} 27-30$ Project ECHO and LC served as parallel implementation strategies for enabling the provision and uptake of evidence-based information. Combining these models leveraged concurrent provider education and clinical assistance through Project ECHO while FQHC staff received training and technical support to implement change via the LC.

Our study builds on this work by providing context about the specific needs and knowledge gaps that FQHC staff identified as barriers and facilitators to delivering better care to their SGM patients during Transforming LGBT Care. The primary aim of this study is to identify factors affecting implementation of the initiative's goals ${ }^{15}$ in order to better categorise potential barriers and facilitators that future implementers may encounter and anticipate their effects on the desired outcomes of their work. We used a general inductive approach to conduct content analysis of transcripts from Project ECHO clinical case presentation, LC meetings and semistructured interviews with FQHC leadership and implementation teams to answer the following research questions ${ }^{1}$ : What clinical practice and health systems and institutional culture factors impacted implementation? and ${ }^{2}$ To what extent did health systems and institutional culture factors act as barriers and facilitators to improving primary care for SGM people? The secondary outcome was clarification of how intervention participants used Project ECHO sessions versus LC meetings to obtain information that influenced implementation of the initiative at their FQHC.

\section{METHODS}

\section{Participants and setting}

Ten FQHCs in rural and urban settings participated in Transforming LGBT Care from March 2016 to March 2017. Participating FQHCs were located throughout the USA and diverse in size, populations served and urbanicity. Detailed characteristics of these health centres have been previously published. ${ }^{15}$ Each FQHC was represented by an implementation team consisting of a QI facilitator, provider champion, and additional clinical and administrative staff who supported the QI facilitator and provider champion. QI facilitators were experienced in programme implementation and were responsible for coordinating and implementing tasks related to the initiative. Provider champions were PCPs responsible for piloting workflow changes with their clinical care teams and gaining clinical staff's buy-in for initiative tasks and goals. Participants' specialties and job roles can be found in table 1.

\begin{tabular}{lc}
\hline Table 1 Participant characteristics & \\
\hline Project ECHO LGBT participants ( $\mathbf{n}=40)$ & $\mathbf{N}(\%)$ \\
\hline Family practice & $27(67.5)$ \\
\hline Internal medicine & $10(25.0)$ \\
Paediatrician & $2(5.0)$ \\
\hline Infectious disease & $1(2.5)$ \\
Provider champions ( $=14)$ & \\
Internal medicine & $8(57.1)$ \\
Family medicine & $5(35.7)$ \\
\hline Paediatrician & $1(7.1)$ \\
Senior leaders ( $\mathrm{n}=21$ ) & \\
\hline Chief clinical officers & $11(52.4)$ \\
Chief executive officer/executive director & $9(42.9)$ \\
Chief operating officer & $1(4.8)$ \\
\hline
\end{tabular}

LGBT, lesbian, gay, bisexual and transgender; Project ECHO, Project Extension for Community Healthcare Outcomes. 


\section{Intervention}

All Project ECHO sessions, LC meetings and interviews were held virtually on a videoconferencing platform (Zoom) between March 2016 and March 2017, with the exception of two in-person LC meetings. The Project ECHO didactic curriculum and LC topic list were previously published. ${ }^{15}$

\section{Data sources}

We conducted secondary analysis of audio recording transcripts from three sources: FQHC staff members' Project ECHO clinical case presentations $(n=64)$; LC meetings attended by FQHC staff; $(n=15)$ and semistructured key informant interviews conducted by lead LC faculty with each FQHC's senior leaders and QI facilitators as part of the LC to debrief the impact of organisational climate and capabilities on implementing clinical and process changes $(n=20)$. ECHO case presentations averaged 28 min long, LC meetings averaged 1 hour 37 min long and interviews averaged 1-hour long.

\section{Qualitative approach and research paradigm}

We conducted qualitative content analysis using an interpretivist approach. ${ }^{31} 32$ We aimed to capture subjective facilitators and barriers from the perspective of participants within their social and structural contexts, in lieu of seeking objective factors that would be universally applicable, as these contexts are integral to understanding the conditions of implementing the initiative. ${ }^{31} 32$ Two major themes emerged: (1) objective discussion of clinical topics surrounding patients' health, wellness and treatment; and (2) health systems and cultural factors identified as part of operation as a healthcare organisation with respect to internal and external customs and social institutions. These themes were chosen to align with the objectives of this study, reflecting our inductive approach. A conceptual content analysis procedure was used to determine the frequency and patterns of subthemes within each major theme. Additionally, this analysis procedure was used to identify the most prevalent barriers and facilitators from the perspective of participants. ${ }^{33-35}$

\section{Data analysis}

To answer our first research question, the research team conducted inductive transcript review to identify influential factors (subthemes) within the two major themes. To ensure clinical subthemes reflected known health disparities among SGM, we deductively derived additional subthemes from a literature review of SGM health disparities and Centers for Disease Control and Prevention clinical partners' expertise. For example, SGM patients are at an increased risk of substance use and abuse. The prevalence of these disparities in the literature is substantial and we believed relevant to provision of care to these populations. For these reasons, substance abuse was deductively derived as an additional subtheme. ${ }^{134}$

Two researchers who were present during Transforming LGBT Care then reviewed and amended the draft subthemes and codebook to ensure accuracy. The full research team finalised and approved the codebook, with inclusion and exclusion criteria, prior to data analysis.

After finalising the codebook, we conducted a content analysis to code transcript data to subthemes. Transcripts were reviewed and coded simultaneously by two researchers who were not present during the initiative (SR and WJ) and one who was present (KWG). Discordance in coding was resolved during biweekly meetings through verbal discussion, and input from an additional researcher who was present during the initiative (LB) was used to break ties.

To answer our second research question, coders applied valences to data within the health systems and institutional cultural factors theme to identify facilitators (positive) and barriers (negative) ${ }^{36} 37$ The same data analysis and discordance resolution processes took place to identify facilitators and barriers. The coding scheme and frequency of codes can be found in online supplemental appendices 1 and 2.

All qualitative analyses were conducted using NVivo (V.12.0, QSR International, Melbourne, Australia).

\section{Subjectivity of coders}

The research team that developed and finalised the codebook included both clinician researchers $(n=3)$ and nonclinician social science researchers $(n=5)$. Several team members had lived experience as SGM and/or members of racial or ethnic minority communities. Five members of the team were involved in conducting Transforming LGBT Care, including two family physicians. All four coders were social science researchers at one of the partner organisations that conducted the study, two of whom were involved in conducting Transforming LGBT Care.

\section{Patient and public involvement \\ No patient or public involved.}

\section{RESULTS}

\section{Content discussed during Project ECHO sessions versus LC meetings}

We sought to obtain a better understanding of the role of the two evidence-based programmes comprising the Transforming LGBT Care intervention. Figure 1 illustrates how intervention participants used Project ECHO sessions vs LC meetings to obtain information that influenced the practice changes they implemented at their FQHCs.

\section{Clinical topics}

Clinical topic discussions reflected gaps in both knowledge about SGM patients' sexual, behavioural and physical health and in self-efficacy to address them. Participants predominantly used LC time to discuss their FQHC's experiences during Transforming LGBT Care, and seldom raised clinical or condition-specific questions 
Topics Discussed During Project ECHO ( $n=64$ sessions)
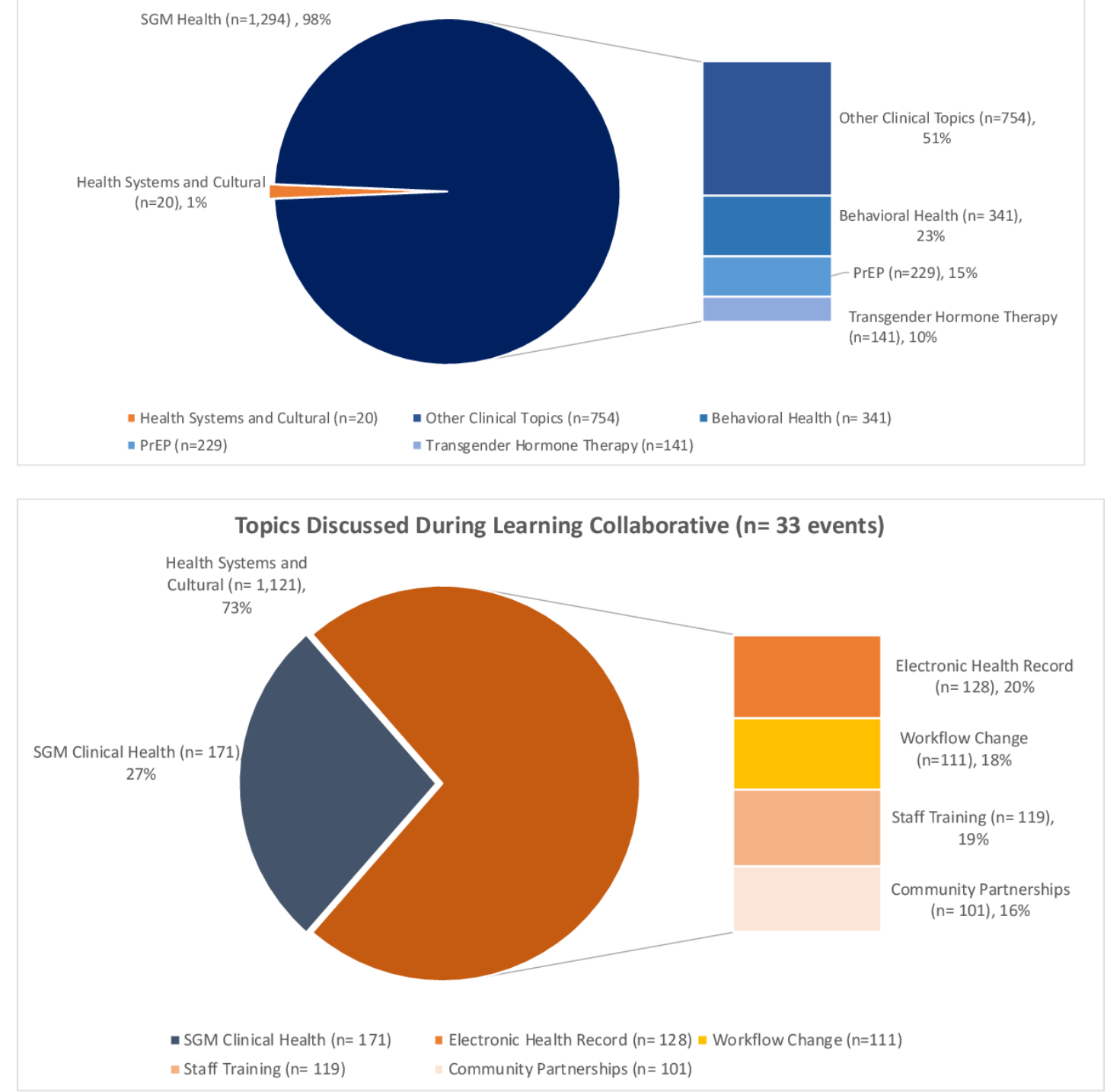

Figure 1 Analysis of 64 Project Extension for Community Healthcare Outcomes (Project ECHO) case presentation transcripts and 33 learning collaborative (LC) session transcripts revealed 1465 unique references to clinical topics related to sexual and gender minority (SGM) clinical health and 1121 unique references to health systems and cultural topics. Clinical topics were predominantly mentioned during Project ECHO case presentations dedicated to clinical knowledge exchange ( $\mathrm{N}=1294)$ vs LC events $(\mathrm{N}=171)$, and were typically evoked in order to share or request objective information or treatment recommendations. The majority of discussion surrounding health systems and cultural topics took place during LC sessions, in the context of supporting programmatic efforts to improve primary care for SGM patients. There were 1101 references to health systems and cultural topics in LC transcripts vs 20 unique references in Project ECHO transcripts. PrEP, pre-exposure prophylaxis

(figure 1). In contrast, ECHO sessions were predominantly used to solicit feedback on specific patient cases or clinical questions (figure 1, table 2).

\section{Barriers and facilitators}

During the process of identifying subthemes under clinical topics, it was observed that, with very few exceptions, clinical topics were part of factual exchanges between Project ECHO case presenters and faculty regarding how to care for one particular patient. Case presenters described the presented patient's medical history and their clinical care, but their stated questions and the ensuing discussion rarely led to them identifying specific health conditions as barriers or facilitators to caring for SGM patients on their panel. For these reasons, clinical topics were not assigned valences for barriers and facilitators.

Health systems and institutional culture topics

Health systems and institutional culture topics encompassed descriptions of healthcare operations and the customs and social institutions both within the FQHC and in the external environment. Reference to how health systems and institutional culture impacted provision of clinical care was notably absent from ECHO sessions, with most discussion of FQHCs' health systems 
Table 2 Clinical topic subthemes, contexts and example quote

\begin{tabular}{|c|c|c|}
\hline Subtheme & Context & Quote \\
\hline Behavioural Health & $\begin{array}{l}\text { The recommendation to be mindful of patients' } \\
\text { diverse care needs was particularly salient } \\
\text { for transgender and gender non-conforming } \\
\text { patients, who frequently had behavioural health } \\
\text { needs that were unmet by behavioural health } \\
\text { services. }\end{array}$ & $\begin{array}{l}\text { "My main questions were how to balance... her mental health, } \\
\text { depression, and alcohol dependence, [and her] uncontrolled } \\
\text { diabetes with the management(of her gender-affirming) } \\
\text { hormones and supporting her in her gender dysphoria." }\end{array}$ \\
\hline HIV PrEP & $\begin{array}{l}\text { Participants frequently sought expert faculty } \\
\text { feedback on prescribing and ensuring } \\
\text { adherence to PrEP for prevention of HIV and } \\
\text { educating patients or addressing misinformation } \\
\text { about PrEP. }\end{array}$ & $\begin{array}{l}\text { "He's been here for about eighteen years [and] is very fearful } \\
\text { of deportation. [He] admits to frequent, anonymous sex, [and } \\
\text { is] unable to negotiate condom [usage]. Over the course of } \\
\text { many visits, we brought up PrEP. At first, he admitted he } \\
\text { wasn't sure about PrEP. He thought he 'wanted HIV.' He had } \\
\text { the misconception that he couldn't be deported if he had HIV. } \\
\text { Ultimately, we did start it after many discussions;(however, at } \\
\text { the follow-up I discovered)he hasn't been on PrEP this whole } \\
\text { time because I only gave him the first three months and he } \\
\text { never [refilled] the prescription. [When] he returns to care, [how } \\
\text { do I] figure out his HIV risk and what are some concrete ways } \\
\text { that I can add some harm reduction here in primary care? } \\
\text { [Additionally), how do I balance reinitiating PrEP, if he wants it, } \\
\text { with his history of poor follow up?" }\end{array}$ \\
\hline
\end{tabular}

Transgender Participating providers most often sought hormone therapy advice about which hormone and dosage was best suited for their patient, given their particular medical needs.

Participants often requested information about how best to counsel their patients receiving hormone therapy. Counselling advice varied; however, common topics included how to appropriately set patients' expectations about timeline, goals and results of hormone therapy and how to manage patients' feelings towards hormone therapy side effects.

\section{Project ECHO Participant: "(Given my patient's alcohol use} disorder and uncontrolled diabetes), I was wondering if I should switch her to transdermal estrogen, hormone-wise."

Project ECHO Faculty: "In terms of her liver health, certainly, estrogen, there's some thinking that maybe you switch to a transdermal vs oral form that that can be easier on her liver, but by far the biggest threat to her liver health and risk of liver failure is related to her alcohol use disorder. The estrogen formulation she's using is quite secondary."

Project ECHO Participant: "I wanted to get peoples' feedback on if [there] is a better androgen blocker [for a transfeminine patient]. [What] if this patient comes back and says, 'I hate this medication, it's not doing it for me'?"

Project ECHO Faculty: "I have patients who are kind of in a similar situation, saying, like, 'it's not working anymore, why is it not working? Let's increase it.' And, now when I do labs, and I'm...regularly checking labs anyways, I think it can be helpful to affirm, like, your estrogen is in a normal range and we don't want to increase it because we don't want it to change to testosterone, we don't want to increase your clot risk any more. You can check her testosterone to reassure her on that as well."

"This is a client that transferred care from another provider. (With that provider, she)did a sexual health questionnaire where she reported having sex in the past 12 months, [with] both men and women, and [used protection] all of the time. So, during my intake with her, I explored [her sexual health questionnaire responses] from the previous provider] and she was very guarded. So, in later sessions, I kept going back to get a little bit more information to really enrich the sense of where she was coming from [and] it turned out [she was] engaged in a number of BDSM and kink communities."

PrEP, pre-exposure prophylaxis; SOGI, sexual orientation and gender identity.

and institutional culture taking place during the LC. (figure 1). About one-fourth of LC discussions focused on facilitators and/or barriers to implementation, including electronic health records (EHRs), the process of workflow change, staff training and community engagement and partnerships. Discussion of health systems and cultural barriers to implementation was infrequent during Project
ECHO case presentations (figure 1), with only eight total mentions of facilitators $(n=4)$ and barriers $(n=4)$.

\section{Barriers}

The EHR was the most commonly identified barrier to implementation. It was mentioned 32 times in the LC meetings and 3 times during ECHO presentations. For 
some FQHCs, a lack of timely EHR vendor or internal information technology support made it challenging to incorporate SOGI questions and data collection fields into the EHR. Doing so required FQHCs to create new fields in their EHR systems or purchase additional applications from their EHR vendor. FQHCs that were able to input SOGI data into their EHR often had difficulty extracting the data for clinical use and analysis. This challenge was described in the following exchange during an interview:

QI Facilitator Site 1: We still have a little bit of a struggle with the data, too, because our EHR system can be a little cumbersome when it comes to data.

Provider Champion Site 1: [Our EHR is] really good for collecting the data but getting the data back out [is] next to impossible, because there's literally like thousands and thousands of options to pick from. And the only support we can get from our EHR product, it's basically, well, trial and error. "Here, try this or try this." And they can't really give us much guidance as to how to actually build the reports.

Clinicians joining Project ECHO also discussed the limitations of EHR data capture and their impact on clinical care. The inability to document a variety of relevant information was a concern for clinicians presenting cases on patients with complex health needs. During a case presentation, a behavioural health provider expressed concern that they may not be able to use the EHR to pass along timely information about a patient's risk to others who care for the patient:

[How] can we document blood play [a specific highrisk sexual health behavior in which blood is integrated into sexual practices]... that seems very relevant to talking about harm reduction, talking about sexual health and safety? [....]I don't have any good answers for how I might have documented better while also protecting her safety in the medical space with other providers and giving more comprehensive information to the next person. So anything that we could [discuss] about documentation would be really helpful for the next time.

This question demonstrates the inability of the presenter's EHR to accommodate thorough documentation of patient sexual behaviours and illustrates the difficulty most of the FQHCs reported in attempting to incorporate sexual risk behaviour screening questions into their health records.

Although EHR functionality in general was identified as a barrier, successful integration of new data collection fields into the EHR allowed implementation teams to extract necessary data, analyse it, correct input errors and missing values, and identify areas for programmatic improvement. ${ }^{25}$ A QI Facilitator cited the benefits of EHR modifications during a monthly LC check-in:
[I] t's been helpful for us to look at the data, especially around the SOGI questions, in contingency tables or crosstabs. Looking at sexual orientation by gender identity, and looking at gender identity by sex at birth, and just seeing how those numbers overlap. And I guess that's been kind of helpful in terms of noticing we have a lot of [missing clinical data].

While implementation teams were able to make advancements toward the integration of data collection in their EHRs, participants reiterated the need for assistance from EHR vendors to meet their data reporting needs. Since FQHCs are required to report on these data as a part of the Health Resources and Services Administration's Uniform Data System measures ${ }_{1}^{10}$ participants stated a desire for increased accountability from EHR vendors to provide low-cost customisable data collection fields. EHR workflow changes (such as modifications to the SOGI data collection process) required leadership buy-in, freedom to collect and use data, and capacity to implement data collection and engage staff to use the data.

\section{Facilitators}

Changes to portions of clinical care workflows outside of the EHR (eg, using Plan-Do-Study-Act (PDSA) cycles $^{38}{ }^{39}$ to refine processes and procedures for staff to collect SOGI and preferred name information in the clinic) improved availability of information to providers caring for SGM patients and increased awareness of available resources, like community partners or support groups. These workflow changes resulted in movement towards the initiative's goal of improving primary care for SGM patients through increased SOGI data collection, risk-based sexual health history taking and STI and HIV screening.

During an interview, a QI Facilitator discussed how workflow change and leadership support made a positive impact on sexual health history screening:

Our CMO, [...] added [sexual health history] into structured data where they ask for social history. The providers just have to click on there and go into the sexual history, and then we have those five questions that are required. And I think it's been very effective. Usually, all our patients give their sexual history.

A majority of implementation teams reported concurrent implementation of various types of staff training to address specific competencies related to the initiative. Staff training provided specific information and education focused on the work of the initiative, and/ or integrated this information into pre-existing training opportunities like employee orientation. Participants cited these trainings as having a positive impact on both processes and outcomes related to the initiative, as they increased awareness and understanding of the ongoing work. During Project ECHO, a participant outlined the 
clinic-wide trainings now offered at their FQHC site to improve delivery of care to their LGBT patients:

We've offered clinic-wide trainings, diversity trainings. We've taken [the training to clinic-wide meetings] so that staff and medical assistants, front desk, providers are welcome to participate. We have offered a couple of transgender hormone therapy classes for providers, specifically. We've offered a lot of [pre exposure prophylaxis (PrEP)] courses. I think seven all together, now, and have a PrEP protocol for providers.

A majority of participants noted a commitment to making these trainings sustainable. An example of this occurred during an LC meeting, when a participant described creating a playbook (instruction manual) for collecting SOGI data:

We put together a playbook. [I]t's basically, a document that we can provide to anyone that gives them the training so that if for some reason they've had the training and they need extra training, or they need to go back and they want to clarify a point, it just gives them a document that delineates every single step of the process for SOGI data collection, how we're using it, and what we're using it for.

Staff training was also used as a tool to encourage acceptance of workflow changes among clinical providers and front-line staff. The following quote is from a clinical provider who mentioned the positive impact of staff training on the culture at their FQHC:

I know [sexual history screening has increased] because we've been talking about it a lot at our clinic recently. [...] [I] t's been a culture shift [at our] clinic and [our FQHC], in general, with more emphasis on the SOGI data collection [and] just doing a lot of trainings with all staff, throughout our clinics, that I think it's more at the forefront of our peoples' minds. Hopefully, increasingly so, we'll be doing better risk assessments as well as just screening, which is kind of what initially happened here.

Community engagement helped increase patients' access, bringing new patients to the FQHCs through increased community awareness of LGBT services being offered, and augmenting the resources FQHCs were able to provide to their SGM patients. During a Project ECHO case presentation, one provider briefly noted how efforts at community engagement resulted in a patient's entrance into care:

[This case pertains to a] transgender female patient who first came to me in October 2015 after meeting me at an outreach event in a neighboring town. I'd gone to speak to a transgender discussion group there and she [was receiving hormone therapy from] an endocrinologist that she no longer feels comfortable with because she was saying he wouldn't draw lab work [and] wasn't open to any change in medication regimens.

Additionally, FQHC staff discussed the benefits of community partnerships with entities such as local health departments, advocacy groups and SGM-specific behavioural health treatment centres. Participants discussed how partners offered financial support, staff training or legal services deemed beneficial to supporting the health and psychosocial needs of their SGM patients. One QI Facilitator discussed how financial assistance from a community partner enabled them to meet a need for transgender patients.

So, we actually secured some funding to provide financial assistance to those clients seeking name change, and we're going to work with a communitybased organization that's offered...Know Your Rights trainings on legal needs of transgender people to collaborate with on some community-based forums and workshops.

FQHCs were also able to hire additional staff (ie, outreach coordinators and pre-exposure prophylaxis (PrEP) Navigators) and conduct appropriate referrals to community agencies focused on quality of care for SGM patients. Community partnerships emerged as a key facilitator to overall capacity to address healthcare disparities for SGM patients. Ultimately, these partnerships were a facilitator not only to FQHCs' ability to provide care, but also to their ability to develop more trusting relationships within the SGM community at large.

\section{Lessons learnt}

Participation in the initiative was not without its own barriers. During LC meetings, staff expressed that tasks required or suggested as part of the initiative were not their only responsibilities. When struggling to make progress and contribute to LC meetings, implementation teams often described that the priority of initiative tasks had fallen relative to their other job duties. Project ECHO took place during clinical hours; therefore, to participate, providers had to be blocked from patient visits during that time. This was not consistently possible and was dependent on the needs of the organisation and its patients.

While EHRs were a barrier across FQHCs, it was observed that organisations that had EHR and data staff were more successful in overcoming challenges to integrating, capturing and extracting data. This was especially pronounced for implementation teams that included an EHR and data staff member who was dedicated to the larger initiative.

In addition to requiring leadership buy-in and usable data, implementation teams also needed to engage patients and other staff to design successful workflow changes. Examples included: (1) conducting focus groups with Spanish-speaking patients to determine how to translate SOGI questions after discovering that the initial 
questions were not comprehensible in Spanish; (2) using (PDSA) cycles with administrative staff to test new intake forms containing SOGI and (3) sending climate surveys to staff and providers who were trained and expected to collect SOGI to gauge their buy-in and challenges. When implementing workflow changes to collect SOGI data, some implementation teams discovered discomfort answering and asking the questions, from patients and staff, respectively. However, the majority of teams stated that they had not received complaints or that complaints were rare.

The staff trainings discussed by implementation teams in LC meetings were often designed by internal FQHC staff. This required passionate, driven staff to prioritise the research and time necessary to create the training. Most staff trainings were implemented as required continuing education for staff, either during new staff onboarding or routine staff meetings. These trainings were predominantly comprehensive of SGM generally; however, some implementation teams offered specific topical training, such as PrEP or transgender health. These trainings were optional for FQHC staff.

\section{DISCUSSION}

These findings support the feasibility of combining LC and Project ECHO to address interrelated components of health system change by providing two different forums for discussion and interaction with experts. We found that Project ECHO clinical learning sessions were used largely for the discussion of clinical issues and the exchange of educational content related to patient care, and that LC meetings, which had a more explicit focus on addressing system-level challenges, were used to discuss barriers and facilitators to using knowledge acquired at Project ECHO to implement recommended practices. While Project ECHO built competency in clinical care delivery through didactic and case presentations, concurrent LC meetings provided forums for participants to focus on health systems, cultural, and programmatic changes needed to improve care for SGM people.

The design of the Transforming LGBT Care initiative created learning systems that were reinforcing over time and across health systems. Enhanced clinical knowledge is an essential element to improve care for SGM patients but can be effectively applied only when appropriate health systems are in place, such as effective SOGI data collection workflows and enhanced EHR functionality. System-level issues, which presented significant barriers to achieving project goals, were essential to address, but required a different forum to facilitate adaptation of clinical recommendations to fit FQHCs' real-world resources and environment.

Our content analysis demonstrates the challenges faced by health centre staff implementing new workflow processes related to improved care for SGM patients. Our findings align with previous research on creating organisational change within a health system through engagement of internal and external stakeholders and integrative implementation, evaluation and adjustment. ${ }^{40}{ }^{41}$ Specifically, clinical care is increasingly delivered by teams of healthcare professionals working together to achieve common goals. Patient care often requires complex changes in processes, workflows and supportive data tools. Developing and implementing these tools requires a diverse team that includes clinicians, administrators and clinical support staff with experience in QI approaches, change management and implementation science. This work indicates that both clinical expertise emphasising knowledge acquisition and QI expertise emphasising staff engagement, data collection and integration, and change management are essential components of improving care. These findings suggest that initiatives focused only on enhancing clinical knowledge may be less successful if the goals of the project require system changes.

This study is not without limitations. To participate in the initiative, FQHCs had to apply, and only those that could demonstrate leadership buy-in to provide staff and resources were selected. Having leadership support at the onset was an influential factor, as FQHCs started with allocated staff and resources and leadership could be contacted when barriers occurred that required leadership attention. Furthermore, available data did not permit pre-post comparison of changes made at the health systems level for individual FQHCs or aggregate analysis of pre-post system-level change for the cohort of 10 FQHCs. Additionally, the semistructured interviews were limited to FQHC leadership and QI facilitators. We were not able to interview staff who were not engaged in the initiative or patients at the individual health centres. As the intention of the interviews were to provide progress updates to FQHC leadership and QI facilitators and gauge individual progress, additional interviews were not within the scope of the QI initiative.

This study provides preliminary evidence for the feasibility of utilising population-based Project ECHO clinics as part of strategies to improve healthcare for vulnerable subpopulations, particularly when combined with an LC to collaborate on making system-level changes. Additionally, this study provides evidence for facilitators and barriers to the implementation of these evidence-based programmes to improve population health. These findings are critical to future efforts to address population health disparities through similar initiatives as they provide a landscape of influential factors to consider during design and implementation. We propose that future work should employ Project ECHO and LC as implementation strategies to facilitate modifications at the system-level to improve provision of care to SGM. As part of this work, evaluation of patient-level outcomes and perspectives should be prioritised to further understand the impact of these efforts. In addition to evaluating the combined effects of these implementation strategies in other healthcare settings, patient-level data will provide a more holistic understanding of these strategies on population health, 
including patients' acceptability of modifications made to address their health needs.

\section{Author affiliations}

${ }^{1}$ Department of Behavioral and Community Health Sciences, University of Pittsburgh, Pittsburgh, Pennsylvania, USA

${ }^{2}$ Weitzman Institute, Community Health Center Inc, Middletown, Connecticut, USA

${ }^{3}$ Center for LGBT Health Research, University of Pittsburgh, Pittsburgh,

Pennsylvania, USA

${ }^{4}$ Division of STD Prevention, Centers for Disease Control and Prevention, Atlanta, Georgia, USA

${ }^{5}$ HIV/AIDS, Hepatitis, STD and TB Administration, District of Columbia Department of Health, Washington, District of Columbia, USA

Acknowledgements We would like to acknowledge and thank Woodlyn Joachim, MPH for her role in data analysis.

Contributors KWG is the guarantor and led development of research question, data analysis, data summary and manuscript preparation. LB assisted with development of research question, data summary and manuscript preparation. SR assisted with development of research question, data analysis, data summary and reviewed drafts of manuscript to provide line edits. BF provided clinical expertise, assisted with development of research question, and reviewed drafts of manuscript to provide line edits. DL provided programme expertise, assisted with development of research question and reviewed drafts of manuscript for approval. DA provided clinical expertise, assisted with development of research question and reviewed drafts of manuscript to provide line edits.

Funding The authors disclosed receipt of the following financial support for the research, authorship, and/or publication of this article: This work was supported by the Centres for Disease Control and Prevention (grant number: 6 NU380T00022305-03); and the National Centre for Advancing Translational Sciences (attributable to KWG, grant number: TL1TR001858).

Competing interests None declared.

Patient consent for publication Not applicable.

Ethics approval The Institutional Review Board at Community Health Centre approved the study protocol and granted an exemption for secondary analysis of data collected during Transforming LGBT Care, which included waiver of written informed consent (IRB ID: 1104).

Provenance and peer review Not commissioned; externally peer reviewed.

Data availability statement Data are available upon reasonable request. Data are available on request to the corresponding author or Weitzman Institute at Community Health Centre.

Supplemental material This content has been supplied by the author(s). It has not been vetted by BMJ Publishing Group Limited (BMJ) and may not have been peer-reviewed. Any opinions or recommendations discussed are solely those of the author(s) and are not endorsed by BMJ. BMJ disclaims all liability and responsibility arising from any reliance placed on the content. Where the content includes any translated material, BMJ does not warrant the accuracy and reliability of the translations (including but not limited to local regulations, clinical guidelines, terminology, drug names and drug dosages), and is not responsible for any error and/or omissions arising from translation and adaptation or otherwise.

Open access This is an open access article distributed in accordance with the Creative Commons Attribution Non Commercial (CC BY-NC 4.0) license, which permits others to distribute, remix, adapt, build upon this work non-commercially, and license their derivative works on different terms, provided the original work is properly cited, appropriate credit is given, any changes made indicated, and the use is non-commercial. See: http://creativecommons.org/licenses/by-nc/4.0/.

\section{ORCID iDs}

Kelly W Gagnon http://orcid.org/0000-0001-7996-8951

Lauren Bifulco http://orcid.org/0000-0002-5759-874X

\section{REFERENCES}

1 Substance Abuse and Mental Health Services Administration. 2018 National Survey on Drug Use and Health. In: Lesbian, Gay, \& Bisexual (LGB) Adults, 2020..
2 Pharr JR, Kachen A, Cross C. Health disparities among sexual gender minority women in the United States: a population-based study. J Community Health 2019;44:721-8.

3 Connolly D, Gilchrist G. Prevalence and correlates of substance use among transgender adults: a systematic review. Addict Behav 2020;111:106544.

4 Parent MC, Arriaga AS, Gobble T, et al. Stress and substance use among sexual and gender minority individuals across the lifespan. Neurobiology of Stress 2019;10:100146.

5 Goldhammer H, Smart AC, Kissock LA, et al. Organizational strategies and inclusive language to build culturally responsive health care environments for Lesbian, gay, bisexual, transgender, and Queer people. J Health Care Poor Underserved 2021;32:18-29.

6 National LGBT Health Education Center. Building patient-centered medical homes for lesbian, gay, bisexual, and transgender patients and families. Fenway Institute, 2016.

7 Unger CA. Care of the Transgender Patient: A Survey of Gynecologists' Current Knowledge and Practice. Journal of Women's Health 2015;24:114-8.

8 Vance SR, Halpern-Felsher BL, Rosenthal SM. Health care providers' comfort with and barriers to care of transgender youth. $J$ Adolesc Health 2015;56:251-3.

9 Haymer M. Transgender patients: prejudice and training needs among trainees in 6 us emergency medicine residency programs, 2014.

10 Grasso C, Goldhammer H, Funk D, et al. Required sexual orientation and gender identity reporting by US health centers: first-year data. Am J Public Health 2019;109:1111-8.

11 Peitzmeier SM, Agénor M, Bernstein IM, et al. "It can promote an existential crisis": factors influencing Pap test acceptability and utilization among transmasculine individuals. Qual Health Res 2017:27:2138-49.

12 Cahill S, Makadon H. Sexual orientation and gender identity data collection in clinical settings and in electronic health records: a key to ending LGBT health disparities. LGBT Health 2014;1:34-41.

13 Hudson KD, Romanelli M. "We Are Powerful People": HealthPromoting Strengths of LGBTQ Communities of Color. Qual Health Res 2020;30:1156-70.

14 Quinn KG, Reed SJ, Dickson-Gomez J, et al. An exploration of syndemic factors that influence engagement in HIV care among black men. Qual Health Res 2018;28:1077-87.

15 Furness BW, Goldhammer H, Montalvo W, et al. Transforming primary care for Lesbian, gay, bisexual, and transgender people: a collaborative quality improvement initiative. Ann Fam Med 2020;18:292-302.

16 Arora S, Kalishman S, Thornton K, et al. Expanding access to hepatitis $C$ virus treatment-Extension for community healthcare outcomes (echo) project: disruptive innovation in specialty care. Hepatology 2010;52:1124-33.

17 Anderson D, Zlateva I, Davis B, et al. Improving pain care with project echo in community health centers. Pain Medicine 2017;18:1882-9.

18 Katzman JG, Comerci G, Boyle JF, et al. Innovative telementoring for pain management: project echo pain. $J$ Contin Educ Health Prof 2014;34:68-75

19 Zhou C, Crawford A, Serhal E, et al. The impact of Project echo on participant and patient outcomes. Academic Medicine 2016;91:1439-61.

20 McBain RK, Sousa JL, Rose AJ, et al. Impact of Project echo models of medical Tele-Education: a systematic review. J Gen Intern Med 2019;34:2842-57.

21 Catic AG, Mattison MLP, Bakaev I, Melissa LP, et al. ECHO-AGE: an innovative model of geriatric care for long-term care residents with dementia and behavioral issues. J Am Med Dir Assoc 2014;15:938-42.

22 Farris G, Sircar M, Bortinger J, et al. Extension for community healthcare Outcomes-Care transitions: enhancing geriatric care transitions through a multidisciplinary Videoconference. J Am Geriatr Soc 2017;65:598-602.

23 Fisher E, Hasselberg M, Conwell Y, et al. Telementoring primary care clinicians to improve geriatric mental health care. Popul Health Manag 2017;20:342-7.

24 Institute for Healthcare Improvement. The breakthrough series: IHI's collaborative model for achieving breakthrough improvement. Diabetes Spectrum 2004;17:97-101.

25 Grasso C, Goldhammer H, Brown RJ, et al. Using sexual orientation and gender identity data in electronic health records to assess for disparities in preventive health screening services. Int J Med Inform 2020;142:104245.

26 National Association of Community Health Centers. Transforming primary care for LGBTQ+ people. Available: https://www.nachc.org/ 
toolkit-transforming-primary-care-for-lgbt-people/ [Accessed 30 Oct 2021].

27 Almazan AN, King D, Grasso C. City-Level structural stigma and patient sexual orientation and gender identity data collection at US health centers, 2018. Am J Public Health 2021.

28 Streed CG, Grasso C, Reisner SL, et al. Sexual orientation and gender identity data collection: clinical and public health importance. Am J Public Health 2020;110:991-3.

29 Ayhan CHB, Bilgin H, Uluman OT, et al. A systematic review of the discrimination against sexual and gender minority in health care settings. Int J Health Serv 2020;50:44-61.

30 Dichter ME, Ogden SN. The challenges presented around collection of patient sexual orientation and gender identity information for reduction of health disparities. Med Care 2019;57:945-8.

31 Alharahsheh $\mathrm{HH}$, Pius A. A review of key paradigms: Positivism vs interpretivism. Glob Acad J Humanit Soc Sci 2020;2:39-43.

32 Goldkuhl G. Pragmatism vs interpretivism in qualitative information systems research. Eur J Inf Syst 2012;21:135-46.

33 Hsieh H-F, Shannon SE. Three approaches to qualitative content analysis. Qual Health Res 2005;15:1277-88.
34 Krippendorff K. Content analysis: an introduction to its methodology. Sage publications, 2018

35 Raskind IG, Shelton RC, Comeau DL, et al. A review of qualitative data analysis practices in health education and health behavior research. Health Educ Behav 2019;46:32-9.

36 Crespi I. Attitude research, 1965.

37 Romano Jr NC, Donovan C, Chen H. A methodology for analyzing web-based qualitative data. J Manage Inf Syst 2003;19:213-46.

38 Coury J, Schneider JL, Rivelli JS, et al. Applying the Plan-Do-StudyAct (PDSA) approach to a large pragmatic study involving safety net clinics. BMC Health Serv Res 2017;17:1-10.

39 Leis JA, Shojania KG. A primer on PDSA: executing plan-do-studyact cycles in practice, not just in name. BMJ Qual Saf 2017;26:572-7.

40 Greene SM, Reid RJ, Larson EB. Implementing the learning health system: from concept to action. Ann Intern Med 2012;157:207-10.

41 Ferlie EB, Shortell SM. Improving the quality of health care in the United Kingdom and the United States: a framework for change. Milbank Q 2001;79:281-315. 